\title{
Transverse magnetic field distribution in the vortex state of noncentrosymmetric superconductor with $\mathrm{O}$ symmetry
}

\author{
Chi-Ken Lu and Sungkit Yip \\ Institute of Physics, Academia Sinica, Nankang, Taipei 115, Taiwan
}

(Dated: October 25, 2018)

\begin{abstract}
We investigate the magnetic field distribution inside a Type II superconductor which has point group symmetry $O$ such as $\mathrm{Li}_{2} \mathrm{Pt}_{3} \mathrm{~B}$. The absence of inversion symmetry as a departure from perfect cubic group $O_{h}$ causes a magnetization collinear with the phase gradient associated with the order parameter, and a component of current collinear with the local magnetic field. In the vortex state, we predict, by solving the Maxwell equation, a local magnetic field transverse to the vortex lines. The probability distribution of this transverse field is also obtained for the prospective muon-spinrotation measurements.
\end{abstract}

PACS numbers: 74.25.Op,74.20.De,74.70.Tx

\section{INTRODUCTION}

Noncentrosymmetric heavy-fermion superconductors have recently triggered much theoretical and experimental attention. The family of $\mathrm{Li}_{2} \mathrm{~B}\left(\mathrm{Pd}_{1-x} \mathrm{Pt}_{x}\right)_{3}$ as well as the compounds $\mathrm{CePt}_{3} \mathrm{Si}$ and $\mathrm{CeRhSi}_{3}$ are among the major examples $, 1,2,3,4,5,6,7,8,9$ The lack of inversion symmetry of background crystal causes an unconventional pairing in the superconducting state and a number of consequences are predicted $\stackrel{10,11,12,13}{1}$ It is commonly believed that in the normal state the Fermi surface is split by the spin-orbital coupling as a result of the broken inversion symmetry, and the pairing wavefunction is in a mixture of singlet and triplet pseudospin state. $\stackrel{\underline{\underline{10}}}{\text { Suggested by the }}$ low temperature measurements of penetration depths, the family of $\mathrm{Li}_{2} \mathrm{~B}\left(\mathrm{Pd}_{1-x} \mathrm{Pt}_{x}\right)_{3}$ with point group symmetry $O$ demonstrates a trend from a conventional gap to a nodal gap caused by mixing with a stronger triplet pairing as the composition $x$ varies from 0 to $1 \stackrel{2}{\underline{2}}$ The conclusion that the $\mathrm{Li}_{2} \mathrm{Pd}_{3} \mathrm{~B}$ compound is a fully gapped superconductor is consistent with other measurements such

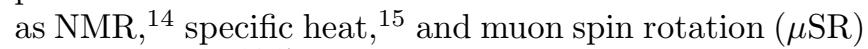
measurements $\frac{16,17}{1}$ The nodal gap structure of $\mathrm{Li}_{2} \mathrm{Pt}_{3} \mathrm{~B}$ hinted by the penetration depth measurement, however, is controversial since the specific heat and $\mu \mathrm{SR}$ measurements suggest conventional pairing. $\stackrel{15,17}{\underline{10}}$

Microscopically, the lack of inversion symmetry allows a parity-breaking spin-orbital interaction which leads to a magnetization induced by the phase gradient associated with the superconducting order parameter, and

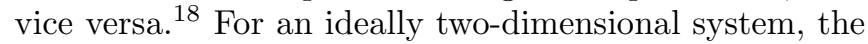
phase gradient induced by the constant magnetic field, or the so-called helical phase, might be measured using a Josephson junction between a conventional and a noncentrosymmetric superconductor ${ }^{\underline{7}}$ However, this scheme is not feasible when considering the decay of magnetic field in a real three-dimensional bulk superconductor. On the other hand, a unique and macroscopic distribution of magnetic field is generated inside the noncentrosymmetric superconductors due to the induced phase graident and magnetization $19,20,21,22$ Therefore, the measurement of the local field distribution, such as the $\mu \mathrm{SR}, \stackrel{23}{\rightleftharpoons}$ can be used to detect the unconventional pairing. Given the fact that these noncentrosymmetric compounds are strong Type II superconductors,$\frac{4.8}{,}$ the effects of lacking inversion symmetry on the vortex state are of importance. In this paper we focus on the noncentrosymmetric superconductors with point group symmetry $O$, such as $\mathrm{Li}_{2} \mathrm{Pt}_{3} \mathrm{~B}$, where a distribution of transverse magnetic field is generated in addition to the ordinary field parallel with the vortex line $\underline{22}$

In Sec II we first derive the magnetic distribution associated with an isolated vortex line. In particular, we note a singular bound current defined by the curl of magnetization appears within the vortex core. It appears because the magnetization here is proportional to the gradient of order parameter phase associated with the vortex state. Hence a new boundary condition is necessary for the azimuthal field to balance the singular bound current, which was missing in our previous study, ${ }^{22}$ and it leads to a significant correction to this field. In Sec III we extend to the lattice and numerically obtain the tranverse field $B_{\perp}(x, y)$ as well as the associated probability distribution $p\left(B_{\perp}\right)$. In Sec IV we connect the present results with the proposed helical phase, and draw the conclusion in the end.

\section{ONE ISOLATED VORTEX LINE}

Inside a type II conventional superconductor in the presence of external magnetic field, the Abrikosov vortex state with a periodic distribution of internal magnetic field is developed when the applied field is larger than $H_{c 1}$, below which a complete Meissner effect occurs, and less than $H_{c 2}$, beyond which the superconductivity only appears on the surface and completely disappear for further increased field. The internal magnetic field inside a conventional superconductor is parallel with the external one, whereas a transverse component is developed inside this noncentrosymmetric superconductor, as will be shown below. Since the internal field for an isolated vortex is essential to the vortex lattice, below we first solve the single vortex case. 
Consider a noncentrosymmetric superconductor with an isolated vortex line along the $\mathrm{z}$ direction. Below the cylindrical coordinate $(\rho, \phi, z)$ is employed. We denote the order parameter associated with the single vortex as $|\psi| e^{i \chi}$ where the phase $\chi=-\phi$, that is the vortex is said to have the winding number of minus one. Besides, the magnitude $|\psi|$ can be viewed as a constant for positions outside the vortex core that roughly has a size of coherence length $\xi$. The internal local magnetic field $\mathbf{B}$ is then determined by the supercurrent $\mathbf{J}$ through the Ampère's law,

$$
\nabla \times \mathbf{B}=4 \pi \nabla \times \mathbf{M}+\frac{4 \pi}{c}(-e) \mathbf{J},
$$

where the first term on the right represents the bound current due to the magnetization $\mathbf{M}$, which is zero for conventional superconductors. The electric charge has been written as $(-e)$. Here the nonzero $\mathbf{M}$ is proportional to the phase gradient, $\stackrel{18,19}{=}$ namely,

$$
4 \pi \mathbf{M}=\frac{\delta}{\lambda^{2}}\left(\mathbf{A}+\frac{c}{2 e} \nabla \chi\right),
$$

where $\lambda$ is the London penetration depth, $\delta$ has the dimension of length, and the small number $\delta / \lambda \equiv \tilde{\kappa} / 2$ depends on the strength of the spin-orbital interaction associated with the lack of inversion symmetry $\underline{\underline{22}}$ On the other hand, the internal magnetic field also induces a contribution to the supercurrent, which together with the ordinary contribution can be written as,

$$
\frac{4 \pi}{c} e \mathbf{J}=\frac{1}{\lambda^{2}}\left(\mathbf{A}+\frac{c}{2 e} \nabla \chi\right)-\frac{\delta}{\lambda^{2}} \mathbf{B},
$$

where $\mathbf{A}$ is the vector potential defining the internal field. Note that $\hbar=1$ for convenience. $\mathbf{M}$ in eq (2) and the last term in eq. (3) exist only if the superconductor is non-centrosymmetric. We note here that ${ }^{22}$ both $1 / \lambda^{2}$ and $\delta / \lambda^{2}$ are proportional to $1-Y(T)$, where $Y(T)$ is the Yosida function, so $\tilde{\kappa}$ is $\propto\left(1-\frac{T}{T_{c}}\right)^{1 / 2}$ near $T_{c}$ and increases with decreasing temperature, saturating to a constant value as $T \rightarrow 0$.

The solutions to Eq. (11) go back to the standard singly quantized vortex when $\tilde{\kappa}$ is set to be zero. The corresponding internal field $B_{z}=B_{z}^{(0)} \equiv \frac{\Phi_{0}}{2 \pi \lambda^{2}} K_{0}(\rho / \lambda)$ where $\Phi_{0}$ is a quantum of flux $\frac{\pi \hbar c}{e}$ and $K_{0}$ is the modified Bessel function of zeroth order. For nonzero $\tilde{\kappa}$, an azimuthal internal field $B_{\phi}$ emerges. In addition, near the core the magnetization contains an azimuthal component proportional to $1 / \rho$ from the phase gradient term in eq. (21). This diverging behavior of $M_{\phi}$ results in a diverging $B_{\phi} \propto 1 / \rho$, which was unfortunately left out in our earlier work ${ }^{22}$ To see this in more detail, we first note that, due to the Meissner effect, we expect that $\mathbf{B}$, the gauge invariant velocity $\left(\nabla \chi+\frac{2 e}{c} \mathbf{A}\right)$ hence also $\mathbf{M}, \mathbf{J}$ all decay to zero as $\rho \rightarrow \infty$. We note that this guarantees the total magnetic flux associated with a singly quantized vortex remains to be $\Phi_{0}$, as $\oint\left(\mathbf{A}+\frac{c}{2 e} \nabla \chi\right) \cdot d \vec{l}=0$ over a large loop encircling the vortex. Integrating eq (11) over a large area enclosing the vortex and using Stokes theorem, we obtain

$$
\oint \mathbf{B} \cdot d \vec{l}-4 \pi \oint \mathbf{M} \cdot d \vec{l}=\frac{4 \pi}{c}(-e) \oint \mathbf{J} \cdot \mathbf{d} \mathbf{S}
$$

hence automatically the condition of zero total transport current along the vortex line, since $B_{\phi}, M_{\phi}$ decays to zero exponentially at large $\rho$.

However, considering eq (4) for a small loop encircling the vortex, we see that for $\rho \rightarrow 0, B_{\phi}=4 \pi M_{\phi}$. Using eq (21) and the fact that the magnetic flux passing through a small loop must necessarily approaches zero when the size of the loop does, we get $4 \pi M_{\phi} \times 2 \pi \rho=-\frac{\delta}{\lambda^{2}} \Phi_{0}$ hence

$$
B_{\phi}(\rho \rightarrow \xi)=-\frac{\tilde{\kappa}}{2 \lambda} \frac{\Phi_{0}}{2 \pi \rho},
$$

This behavior can also be obtained directly by substituting eq (2) and (3) into (11) and using $\nabla \times \nabla \chi=$ $-2 \pi \delta^{(2)}(\vec{\rho}) \hat{z}$. We get

$$
\nabla \times \mathbf{B}=\frac{4 \pi}{c}(-e) \mathbf{J}+\frac{\tilde{\kappa}}{2 \lambda}\left[\mathbf{B}-\Phi \delta^{(2)}(\vec{\rho}) \hat{z}\right] .
$$

The delta function can be taken care of by an appropriate boundary condition associated with the azimuthal field $B_{\phi}$ at the origin, as in eq (5).

Eq. (6) can be solved in the manner of perturbation in $\tilde{\kappa}$. For $\rho \neq 0$, the delta function can be ignored and the first-order correction $B_{\phi}^{(1)}$ satisfies the following equation, obtained by taking the curl of eq. (6) and using eq. (3),

$$
\left[\frac{d}{d \rho}\left(\frac{1}{\rho} \frac{d}{d \rho} \rho\right)-\frac{1}{\lambda^{2}}\right] B_{\phi}^{(1)}(\rho)=\frac{\tilde{\kappa}}{\lambda} \frac{d}{d \rho} B_{z}^{(0)},
$$

in which the source term on the RHS arises from the extra supercurrent induced by the unperturbed internal field $B_{z}^{(0)}$ as well as the second term in eq. (6). This equation is identical to eq. (37) in our previous work ${ }^{22}$ There the inhomogeneous solution satisfying $B_{\phi} \rightarrow 0$ near the origin was obtained and we shall denote it as $\frac{\tilde{\kappa} \Phi_{0}}{2 \pi \lambda^{2}} f(x)$ with the argument $x$ defined by $x=\rho / \lambda$. (Thus $f(x)$ represents the right hand side of eq (41) in Ref. 22). We note that this inhomogeneous solution does not satisfy the boundary condition in eq. (5), which can be fixed by adding an appropriate homogeneous solution to eq. (7) satisfying eq (5). Since $K_{1}(x)$ fulfills eq. (7) in the absence of the source term and $K_{1}(x) \propto 1 / x$ for small $x$, we can easily write down the desired azimuthal field as,

$$
B_{\phi}^{(1)}(x)=\frac{\tilde{\kappa} \Phi}{2 \pi \lambda^{2}}\left[f(x)-\frac{1}{2} K_{1}(x)\right] .
$$


The azimuthal field still decays exponentially at infinity, justifying the previous assumptions. However, in contrast to our previous results, $\stackrel{22}{\stackrel{2}{2}}$ due to the $K_{1}$ term the azimuthal field for an isolated vortex line now has increasing magnitude towards the core.

\section{LATTICE OF VORTEX LINES}

Since those heavy-fermion superconductors usually have low transition temperatures and large GinzburgLandau parameter, $H_{c 1}$ is practically small and usually much less than the external magnetic field, which causes the superconductor to be in the vortex lattice state in most cases. The components for transverse magnetic fields $B_{x}(x, y)$ and $B_{y}(x, y)$ inside the superconductor are given by the vector sums of eq. (8) over the vortex lines located at each of the lattice vertex. For the family of $\mathrm{Li}_{2} \mathrm{~B}\left(\mathrm{Pd}_{1-x} \mathrm{Pt}_{x}\right)_{3}$, the ratio of $\lambda / \xi$ is about $\underline{\underline{4}} 20$ independent of the Pt composition. The transverse field depends on the lattice geometry, and $d / \lambda$ is the only parameter with $d$ being the lattice constant. Figs. 1 and 2 show the contours of transverse field $B_{\perp}=\sqrt{B_{x}^{2}+B_{y}^{2}}$ in unit of $B_{0}=\tilde{\kappa} \Phi_{0} / 2 \pi \lambda^{2}$ with $d / \lambda=0.5$ for a square and triangular lattices, respectively. Due to the delta function in eq. (6), the regions close to the core have larger transverse fields. The destructive interferences from neighboring vortex lines occur at the middle points on each side and at the center of each square and triangle, respectively.

Statistics on the local internal field $B_{\perp}(x, y)$ give the probability distribution $p\left(B_{\perp} / B_{0}\right)$. In Fig. 3 and Fig. 4. we obtain the probability distributions $p\left(B_{\perp} / B_{0}\right)$ for various values of $d / \lambda$ in the square and triangular lattices, respectively. $p\left(B_{\perp} / B_{0}\right)$ vanishes for $B_{\perp} \rightarrow 0$, and rises up linearly with $B_{\perp}$ for small $B_{\perp}$. It reaches a peak corresponding to the longest contour in Fig. 1 or 2. then decreases with increasing $B_{\perp}$. As the value of $d / \lambda$ decreases, the vortex lines are getting denser and the magnetic fields associated with the peak of $p\left(B_{\perp}\right)$ become greater. One should note, however, that there is a cutoff magnetic field associated with the vortex core for each value of $d / \lambda$ because the minimum length scale for the above theory to be applicable here is the coherence length $\xi$. The colored arrows are used to label these cutoffs for various $d / \lambda$. That is, the values of $B_{\perp}$ beyond these arrows are un-physical and the actual values are expected to be much smaller. We note that the cutoff for a single vortex is approximately given by $\frac{1}{2} K_{1}(\xi / \lambda) \approx 10$ times $B_{0}$. For low density of vortices, i.e. $d / \lambda \gg 1$, the cutoff remains the same as that of a single vortex. For higher density cases, these cutoffs are modified due to the neighboring vortices.

The distributions $p\left(B_{\perp}\right)$ can in principle be obtained from $\mu \mathrm{SR}$ measurements, operated in the following manner. Positive muons with spin polarizing along the external field and hence the vortex lines are injected into the superconductor in the vortex state. In such a set-up for conventional superconductors, the muon spin polarization is not affected by the local magnetic fields, as they are parallel (assuming that the vortex lines are straight, c.f. below). The decay product, the positrons, has an anisotropic distribution, being preferentially along the initial muon spin. However, for our non-centrosymmetric superconductor, the muon spins will precess around the local field $B_{\perp}(x, y)$. This gives rise to an extra decay or oscillation of the anisotropic positron distribution. This distribution is further a function of external field and temperature, with magnetic field magnitude increasing with decreasing temperature if below $T_{c}$. These features may be used to distinguish from other contributions. Thermal fluctuation of vortex lines may also give rise to horizontal fields, but they would increase instead of decrease with temperature. Magnetic fields from nuclear moments would have a temperature dependence uncorrelated with $T_{c}$.

The values of $d / \lambda$ correspond to average external magnetic fields $B_{a v}$ according to the relation $B_{a v}=\frac{2 \pi}{\eta} \frac{\lambda^{2}}{d^{2}} \frac{\Phi_{0}}{2 \pi \lambda^{2}}$, where $\eta$ is a factor depending on the lattice geometry. For square lattice $\eta=1$, and for triangular lattice $\eta=\frac{\sqrt{3}}{2}$. The magnetic field $\frac{\Phi_{0}}{2 \pi \lambda^{2}}$ is about 25 Gauss given $\lambda(T \rightarrow 0)=360 \mathrm{~nm}$ in $\mathrm{Li}_{2} \mathrm{Pt}_{3} \mathrm{~B} \stackrel{\underline{4}}{\underline{4}}$ If the parameter $\tilde{\kappa}$ exceeds $10^{-2}$, then the typical fields according to Fig 3 and 4 will be of order 1 gauss, which is supposed to be measurable in the $\mu \mathrm{SR}$ measurement $\stackrel{24,25}{ }$ In the compound $\mathrm{Li}_{2} \mathrm{Pt}_{3} \mathrm{~B}$, however, $\tilde{\kappa}$ may unfortunately be of order $10^{-3}$ only, with transverse fields that are hard to detect. Nevertheless, our calculations apply to any noncentrosymmetric superconductor with O symmetry. The transverse fields may be easily measurable if $\tilde{\kappa}$ is a few times larger and/or $\lambda$ a few times shorter.

\section{DISCUSSION}

The above paragraphs demonstrate the transverse magnetic field in the vortex state as a signature of broken inversion symmetry. Here we elaborate on the general phase gradient $\vec{Q}=\nabla \chi+\frac{2 e}{c} \mathbf{A}$ since this vector is associated with the helical phase which is described by an order parameter $|\Psi| e^{i \vec{Q} \cdot \vec{r}}$. In a strictly two-dimensional system with open boundary, the phase gradient can be obtained by directly setting the current density in eq. (3) to be zero. Since there is no Meissner effect in such an ideal system, $\vec{Q}$ is determined solely by the external field and was argued to be measurable by a Josephson junction between a conventional and a noncentrosymmetric superconductor $\underline{\underline{?}}$

For our three-dimensional superconductor, $\vec{Q}$ can be obtained from eq. (11) and (3) given the internal magnetic field B. For positions not exactly at the vortex cores,

$$
\frac{c}{2 e} \vec{Q}=\lambda \tilde{\kappa} \mathbf{B}-\lambda^{2}(\nabla \times \mathbf{B}) .
$$


In a real bulk superconductor, it can be seen that the phase gradient is small and not appreciable due to the Meissner effect. For example, consider a noncentrosymmetric superconductor occupied by a single vortex. The unique phase gradient $Q_{z}$ is along the line. Given the internal fields $B_{z}^{(0)}$ and $B_{\phi}^{(1)}$ obtained previously, $Q_{z}$ is vanishingly small at positions $\rho \gg \lambda$. Only near the vortex $Q_{z}$ is large and given by $\frac{\widetilde{\kappa} \ln x}{2 \lambda}$ for $\rho \ll \lambda$. We note however that, at these distances, $Q_{\phi} \rightarrow-\frac{1}{\rho}$, hence $\left|Q_{\phi}\right| \gg\left|Q_{z}\right|$. The phase gradient associated with the helical phase in this noncentrosymmetric superconductors has also been obtained beyond the present modified London theory ${ }^{13}$ (see $\left.\operatorname{also}^{7}\right)$.

\section{CONCLUSION}

We consider the effects of broken inversion symmetry noncentrosymmetric superconductor with $\mathrm{O}$ symmetry such as $\mathrm{Li}_{2} \mathrm{Pt}_{3} \mathrm{~B}$ by calculating the transverse magnetic field in the vortex state. The probability distribution of the field is also calculated for the prospective $\mu \mathrm{SR}$ experiments as a signature of the broken inversion symmetry.

\section{Acknowledgment}

We thank Rustem Khasanov and Baruch Rosenstein useful correspondences. This work is supported by the National Science Council of Taiwan, R.O.C. under grant No.NSC95-2112-M001-054-MY3.
${ }^{1}$ K. Togano, P. Badica, Y. Nakamori, S. Orimo, H. Takeya, and K. Hirata, Phys. Rev. Lett. 93, 247004 (2004).

${ }^{2}$ H. Q. Yuan, D. F. Agterberg, N. Hayashi, P. Badica, D. Vandervelde, K. Togano, M. Sigrist, and M. B. Salamon, Phys. Rev. Lett. 97, 017006 (2006).

3 M. Nishiyama, Y. Inada, and G. Q. Zheng, Phy. Rev. Lett. 98, 047002 (2007)

${ }^{4}$ For a review on $\mathrm{Li}_{2} \mathrm{~B}\left(\mathrm{Pd}_{1-x} \mathrm{Pt}_{x}\right)_{3}$, see P. Badica, T. Kondo, and K. Togano, J. Phys. Soc. Jpn. 74, 1014 (2005).

${ }^{5}$ E. Bauer, G. Hilscher, H. Michor, Ch. Paul, E. W. Scheidt, A. Gribanov, Yu. Seropegin, H. Noel, M. Sigrist, and P. Rogl, Phys. Rev. Lett. 92, 027003 (2004).

6 T. Yasuda, H. Shishido, T. Ueda, S. Hashimoto, R. Settai, T. Takeuchi, T. D. Matusda, Y. Haga, and Y. Ōnuki, J. Phys. Soc. Jpn. 73, 1657 (2004).

7 R. P. Kaur, D. F. Agterberg, and M. Sigrist, Phys. Rev. Lett. 94, 137002 (2005).

${ }^{8}$ For a review on $\mathrm{CePt}_{3} \mathrm{Si}$, see E. Bauer, H. Kaldarar, A. Prokofiev, E. Royanian, A. Amato, J. Sereni, W. BrämerEscamilla, and I. Bonalde, J. Phys. Soc. Jpn. 76, 051009 (2007).

9 R. Settai, T. Takeuchi, and Y. Ōnuki, J. Phys. Soc. Jpn. 76, 051003 (2007).

10 L. P. Gor'kov and E. I. Rashba, Phys. Rev. Lett. 87, 037004 (2001).

11 P. A. Frigeri, D. F. Agterberg, I. Milant, and M. Sigrist, Eur. Phys. J. 54, 435 (2006).

12 D. F. Agterberg and R. P. Kaur, Phys. Rev. B 75, 064511 (2007).

13 K. V. Samokhin, arXiv:0805.3338v1

14 M. Nishiyama, Y. Inada, and G.-Q. Zheng, Phys. Rev. B 71, 220505(R) (2005).

${ }^{15}$ H. Takeya, K. Hirata, K. Yamaura, K. Togano, M. El Massalami, R. Rapp, F. A. Chaves, and B. Ouladdiaf, Phys. Rev. B 72, 104506 (2005).

16 R. Khasanov, I. L. Landau, C. Baines, F. La Mattina, A. Maisuradze, K. Togano, and H. Keller, Phys. Rev. B 73, 214528 (2006).
17 P. S. Häfliger, R. Khasanov, R. Lortz, A. Petrović, K. Togano, C. Baines, B. Graneli, and H. Keller, arXiv:0709.3777 1

18 V. M. Edelstein, Zh. Eksp. Teor. Fiz. 95, 2151 (1989) [Sov. Phys. JETP 68, 1244 (1989)].

19 L. S. Levitov, Yu. V. Nazarov, and G. M. Eliashberg, JETP Lett. 41, 445 (1985).

20 S. K. Yip, J. Low Temp. Phys. 140, 67 (2005).

21 M. Oka, M. Ichioka, and K. Machida, Phys. Rev. B 73, 214509 (2006).

22 C. K. Lu and S. K. Yip, Phys. Rev. B 77, 054515 (2008).

23 S. Lee, in Muon Science: Muons in Physics, Chemistry, and Materials, edited by S. H. Lee et al. (Institute of Physics Publishing, Bristol, 1999).

24 Rustem Khasanov, private communication.

25 As a comparison, A. Schenck in ${ }^{23}$ gives $0.5 \mathrm{G}$ as the resolution limit for measurement of small magnetic moments in heavy fermion systems. 


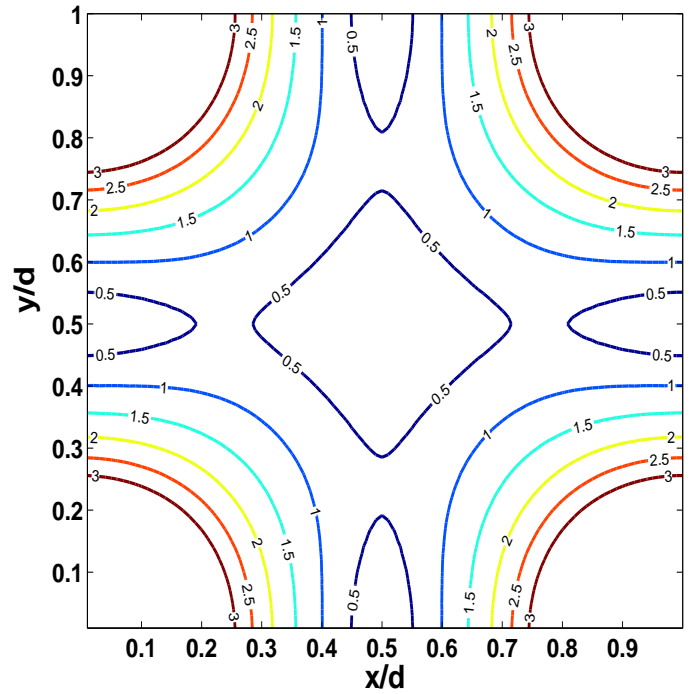

FIG. 1: Contour plot of transverse magnetic field magnitude $B_{\perp}$ for a square lattice. The unit of $B_{\perp}$ is $B_{0}=\tilde{\kappa} \Phi / 2 \pi \lambda^{2}$. The distance $d$ between neighboring lines to the penetration depth $\lambda$ is 0.5 .

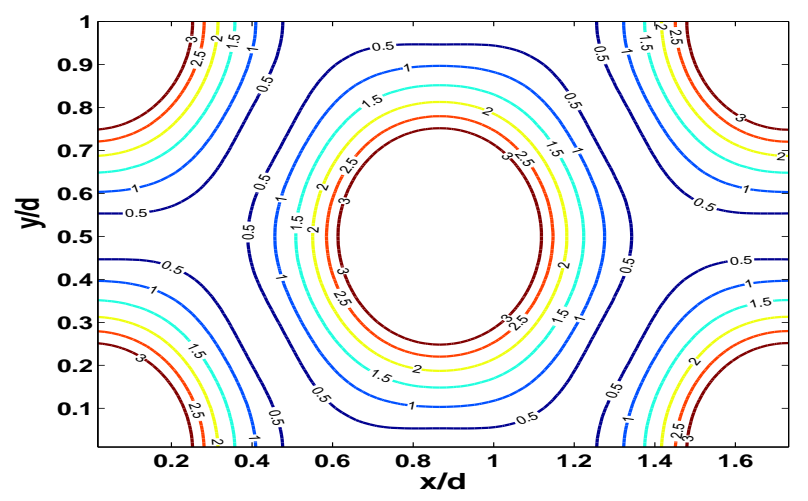

FIG. 2: The same contour plot as Fig. 1 but for a triangular lattice with the same value of $d / \lambda$. 


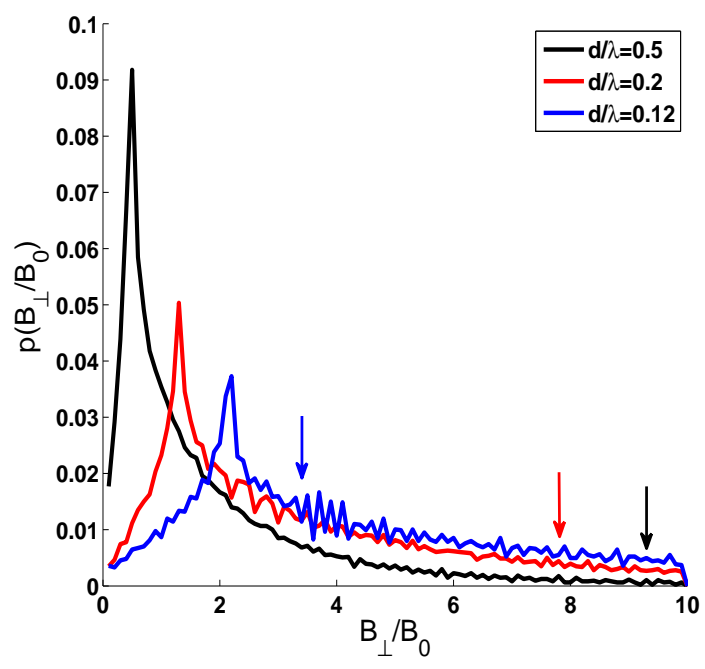

FIG. 3: The probability distribution $p\left(B_{\perp} / B_{0}\right)$ of internal transverse fields for square lattice with various values of $d / \lambda$. The arrows indicate the cutoff magnetic fields associated with the vortex core. The respective cutoff values are 9.6, 7.9, and $3.5 \mathrm{~B}_{0}$.

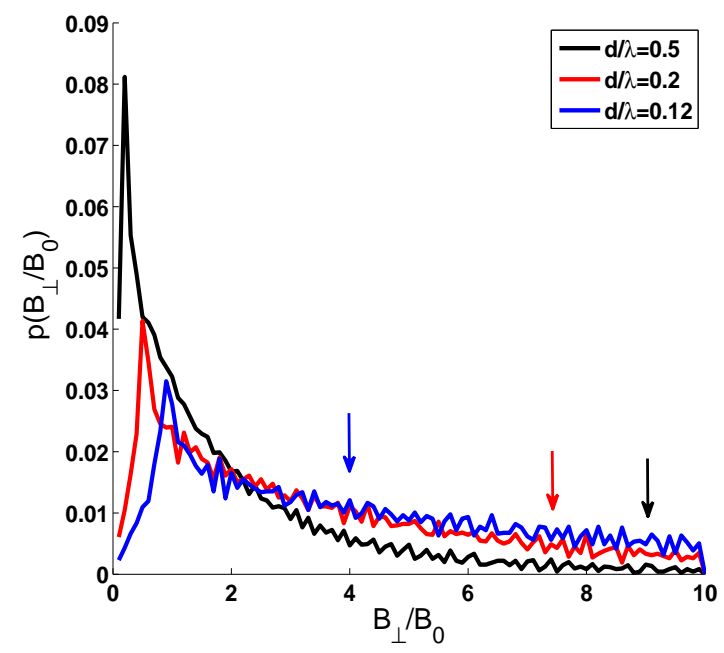

FIG. 4: $p\left(B_{\perp} / B_{0}\right)$ for triangular lattice for various values of $d / \lambda$. The respective cutoff values are $9.2,7.7$, and $4.0 \mathrm{~B}_{0}$. 